\title{
Characterization of Impaired Ventricular Repolarization by Quantification of QT Delayed Response to Heart Rate Changes in Stress Test
}

\author{
Cristina Pérez ${ }^{1}$, Esther Pueyo $^{1,2}$, Juan Pablo Martínez $^{1,2}$, Jari Viik $^{3}$, Pablo Laguna ${ }^{1,2}$ \\ ${ }^{1}$ BSICoS Group, I3A, IIS Aragón, University of Zaragoza, Zaragoza, Spain \\ ${ }^{2}$ CIBER-Bioengineering, Biomaterials and Nanomedicine (CIBER BBN), Madrid, Spain \\ ${ }^{3}$ Faculty of Medicine and Health Techonology, Tampere University of Technology, Tampere, Finland
}

\begin{abstract}
Increased spatio-temporal heterogeneity in ventricular repolarization is related to cardiac instabilities. Slowed adaptation of the QT interval to sudden abrupt changes in heart rate (HR), measured by the time constant of a firstorder-like step response, has been identified as a marker of arrhythmic risk. Since abrupt HR changes are difficult to induce in patients, ramp-like HR variations observed in exercise stress test are considered in this work. The first-order-system time constant is estimated as the delay between the QT series response and the series of memoryless expected $Q T$ series from the instantaneous $H R$, obtained from a fitted hyperbolic regression model. The delay was estimated by minimizing the mean square error separately in the exercise and recovery phases. We applied this procedure to a subset of 251 patients of Tampere university hospital with different risk levels for Coronary Artery Disease (110 nonCAD, 39 low-risk, 12 mild-risk, 90 highrisk). The time constant values ranged from $17 \mathrm{~s}$ to $49 \mathrm{~s}$, which are comparable with the time lags reported in other studies in response to step-like HR changes. The delays in exercise and recovery phases and the difference between these delays are markers with capacity for CAD risk stratification.
\end{abstract}

\section{Introduction}

In recent years, different biomarkers for stratification of patients according to their risk of suffering from ventricular arrhythmias and Sudden Cardiac Death (SCD) have been proposed. Concretely, those reflecting spatiotemporal repolarization dispersion are pivotal for SCD prediction [1]. Intrinsic electrophysiological heterogeneities in the ventricular myocardium lead to ventricular repolarization dispersion, which can be exacerbated in response to changes in heart rate (HR) due to the fact that different ventricular cells present distinct patterns of repolarization adaptation to the HR changes. During these transient situations in which there is an increase in ventricular repolarization dispersion, the vulnerability to the appearance of ventricular arrhythmias can be enhanced in some patients. The adaptation time of the QT interval to sudden changes in HR has been identified as a biomarker for arrhythmic risk [2], with the underlying mechanisms subsequently elucidated by experimental and simulated electrophysiological studies [3]. This phenomenon occurs on top of beat-to-beat variability, commonly quantified under stationary conditions, and can provide complementary information [4]. Previous studies have highlighted the importance of determining normal and abnormal ranges of QT adaptation dynamics in response to sudden changes in HR as a possible way to characterize the risk for cardiac arrhythmias and SCD [5].

However, achieving adaptation time lags from abrupt HR changes are not always easily observed in Holter recordings. Alternatively, ramp-like HR changes can be more feasibly obtained from stress test recordings. The ramp-response of a first-order system is characterized by the same time constant as the step-response. Its characterization would hypothetically provide the same clinical information [6]. The advantage is ramp-like inputs are typically observed in exercise stress tests, where the cardiac system is subject to an approximately linear HR input both during the exercise and recovery phases of the test.

\section{Methods}

\subsection{Database}

This study evaluated stress test $500 \mathrm{~Hz}$-sampled ECG recordings from 251 patients of Tampere university hospital using a bicycle ergometer, targeted to characterize patients with high risk of cardiovascular morbidity and mortality The patients were divided into four groups. The first group, nonCAD, was based on clinical history information and ECG, and the others also were based on coronary angiography results, according to their likelihood for Coro- 
nary Artery Disease (CAD): low-, mild- and high-risk.

\subsection{Signal Preprocessing}

First, a spatially transformed lead derived from Principal Component Analysis (PCA) [8] over the 8 independent standard leads was obtained. Subsequently, a waveletbased algorithm [9] was used to extract both $Q T(i)$ and $R R(i)$ intervals series from each $i$ th beat, combined with a spline interpolation to account for missing $\mathrm{T}$ end values. Outlier values of both $R R(i)$ and $Q T(i)$ series, identified as those deviating more than $\pm 5 \%$ from the running median of each series, computed over 50 beats, were replaced with the corresponding median value. Subsequently, $R R(i)$ and $Q T(i)$ series were interpolated to $4 \mathrm{~Hz}$ to have uniformly sampled $R R(n)$ and $Q T(n)$ time series.

\section{3. $\quad$ Expected HR-dependent QT series}

The first step consists in estimating the $\widehat{Q T}(n)$ series containing the QT values that would correspond to each $R R(n)$ value if the HR was stationary. This series will be the reference to estimate the delay of the observed QT series. To estimate this memoryless expected HR-dependent QT interval series, $\widehat{Q T}(n)$, the values of the parameters $\alpha$ and $\beta$ of different regression models were obtained by fitting $[Q T(n), R R(n)]$ data pairs from three windows: at onset (40 seconds), at the stress peak (20 seconds) and at end (40 seconds) of the stress test, which are marked in Fig.1a and assumed to be stationary and representative of the subject QT-to-RR dependency. The window at the stress peak was replicated twice to have equal weight from each area. The four considered regression models were parabolic, linear, hyperbolic and logarithmic.

The assumption of stationarity at the stress peak is questionable, but it is included to account for the whole excursion of RR when evaluating the RR-to-QT dependency. Given that this window is symmetric around the peak, including ascending and descending HR phases, a compensation for the dynamics is expected, so the mean QT-to-RR relationship should not be far from the stationary one.

\subsection{Time lag estimation}

The time lag between estimated $\widehat{Q T}(n)$ and real $Q T(n)$ time series (example in Fig.1b) was estimated by a Mean Square Error (MSE) fit between the $Q T(n)$ ramp and the $\widehat{Q T}\left(n-\tau^{*}\right)$ ramp, being $\tau^{*}$ the delay providing the minimum MSE. This fitting was performed separately in two phases: $\tau_{e}$ estimated in exercise, and $\tau_{r}$ in recovery, using the marked intervals in Fig.1b (from exercise onset, $n=n_{e, o}$, to exercise end, $n=n_{e, e}$, and from recovery onset, $n=n_{r, o}$, to recovery end, $n=n_{r, e}$ ).

\subsubsection{Delimitation of exercise and recovery ramps}

The exercise onset, $n_{e, o}$ (and the recovery end, $n_{r, e}$ ), are defined as the points with lower significant differences between their preceding linearly adjusted plateau (incline), and the subsequent linearly adjusted incline (plateau) of $\widehat{Q T}(n)$ series, respectively. This search for $n_{e, o}$ is implemented by minimizing the following residual sum [10]:

$$
n_{\{e, o\}}=\arg \min _{k}(J(k))+60
$$

where

$J(k)=\sum_{n=m}^{k-1}\left(\widehat{Q T}(n)-f_{p}(n)\right)^{2}+\sum_{n=k}^{N}\left(\widehat{Q T}(n)-f_{s}(n)\right)^{2}$.

Both, $f_{p}(n)=a_{p}+b_{p} n$ and $f_{s}(n)=a_{s}+b_{s} n$, are the linear estimates of the $\widehat{Q T}(n)$ series preceding and subsequent to point $k$, respectively, being $m=1$ and $N$ equal to the sample at series stress peak. Analogously, for $n_{r, e}$ the same minimization is performed, but now $m$ is equal to the stress peak value and $N$ is the final $\widehat{Q T}(n)$ sample. The $n_{e, o}$ point is delayed (and the $n_{r, e}$ point is advanced) by 60 samples to avoid an error in to estimate the delay due to unpredictable artefacts.

To mark the end of exercise ramp, $n_{e, e}$, firstly the data of both $\widehat{Q T}(n)$ and $Q T(n)$ series from $n_{e, o}$ and the sample $n$ when $Q T(n)=\widehat{Q T}\left(n_{e, o}\right)$, respectively, to the stress peak sample are adjusted by a linear regression model, since their tendency follows a ramp. Then, $n_{e, e}$ is defined as $n_{e, o}+0.65\left(n_{e, c}-n_{e, o}\right)$, being $n_{e, c}$ the point where these adjusted lines get closer. In a similar way, the onset sample of recovery ramp, $n_{r, o}$, was calculated as $n_{r, c}+0.45\left(n_{r, e}-n_{r, c}\right)$, being $n_{r, c}$ the point where the adjusted lines get closer in the recovery phase (Fig.1b). The threshold values 0.65 and 0.45 are chosen as those which maximize the significance in separation of CAD risk groups.

\section{Results}

The lowest root mean square error $\left(\varepsilon_{\mathrm{rms}}\right)$, used to evaluate the goodness of fit the of the QT-to-RR relationship in all groups, was obtained with the hyperbolic model. Therefore, we only analyzed results for this model. Table 1 shows mean and standard deviation of $\alpha$ and $\beta$ values estimated to generate the expected $\widehat{Q T}(n)$ series. A moderate degree of inter-individual variability can be observed in the values of the parameter $\alpha$ across the subjects of each group. Moreover, the mean of the HR values selected for each windows are also given in Table 1. The maximum $\mathrm{HR}$ mean value $\left(\mathrm{HR}_{W_{2}}\right)$ increases considerably as the risk of suffering $\mathrm{CAD}$ decreases. 

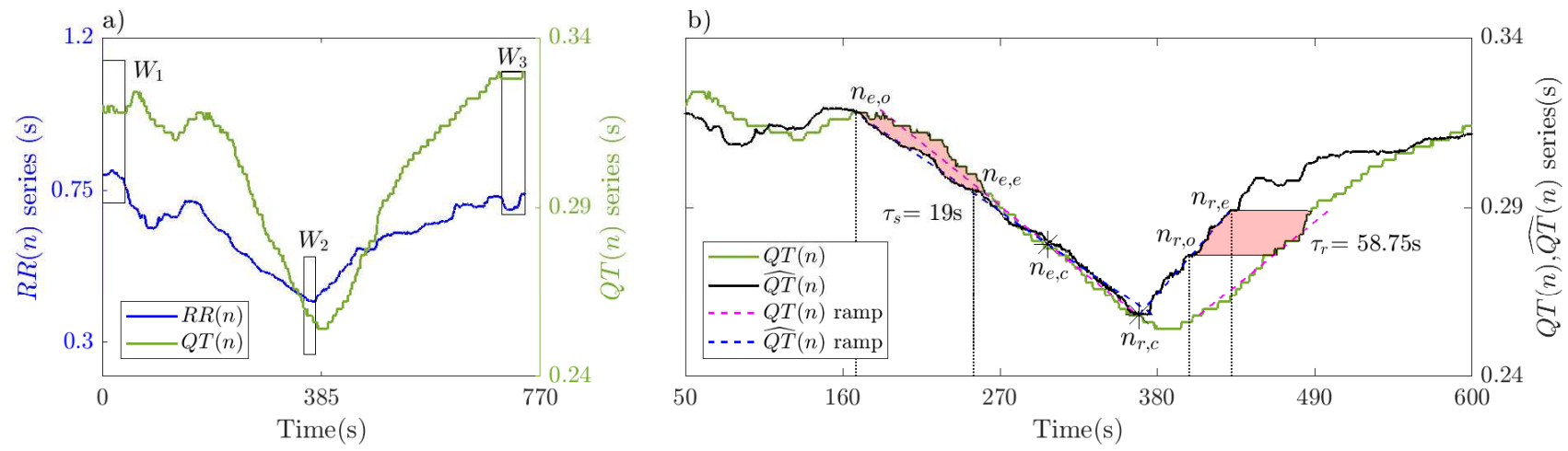

Figure 1. a) The three windows $\left(W_{1}, W_{2}, W_{3}\right)$ were used by estimate the hyperbolic model parameters to calculate the expected HR-dependent QT time series. b) An example of ramps onsets and ends during exercise $\left(n_{e, o}, n_{e, e}\right)$ and recovery $\left(n_{r, o}, n_{r, e}\right)$, and their corresponding delay obtained by the MSE criteria between $Q T(n)$ and $\widehat{Q T}\left(n-\tau^{*}\right)$.

The estimated delay values from the $\widehat{Q T}(n)$ and $Q T(n)$ time series are represented in Fig.2. The $\bar{\tau}_{e}$ delay was clearly shorter in the nonCAD and low-risk groups compared to mild- $(p=0.01, p<0.01)$ and high-risk $(p<0.01, p<0.01)$, being in agreement with the hypothesis that slowed adaptation relates to increased cardiac risk, although the mean exercise delay value of low-risk group is lightly smaller than this value for nonCAD group. In addition, the difference in adaptation time between exercise and recovery was larger in nonCAD and low-risk patients than in mild- ( $p<0.01, p=0.01)$ and high-risk ( $p<0.01, p<0.01)$, suggesting $\Delta_{\tau}=\tau_{r}-\tau_{e}$ could be a risk biomarker. A slight shortening was noted during the recovery in mild-risk group, behaving contrary to exercise.

\section{Discussion}

When we analyzed the $\widehat{Q T}(n)$ and $Q T(n)$ series, we realized they became overlapped, with no significant delay time when approaching the stress peak in the exercise phase (Fig.1b). This phenomenon is in agreement with recent findings [11] showing that the time for ventricular repolarization adaptation to sympathetic provocation becomes progressively reduced for increasingly higher levels of beta-adrenoceptors' stimulation, as occurs when approaching the stress peak. Electrophysiological simulations including changes in Autonomic Nervous System (ANS) and in HR as those studied here could shed light on this observed behavior. To avoid including this area with marked overlap, where the system is clearly different to the case with regular ANS action, the exercise and recovery delays were computed considering only parts of exercise and recovery phases distant to the stress peak. The time delays in exercise and in recovery seem to be very similar for nonCAD and low-risk groups, and for mild- and high-risk groups. So these biomarkers might only work to indicate minor or elevated risk of suffering CAD.

The mean time lag values (Fig.2) are of the same order of magnitude as those reported in [2], where the time constants estimated from selected step-like response reached values ranging from 36 to 58 seconds, depending on the studied patient subgroup. This is compatible with the hypothesis that time lag, measured as proposed here, at the exercise and recovery ramps or in a sudden step-like HR change protocol can be equivalent. Moreover, patients of [2] were survivors of acute myocardial infarction while the common characteristic in this study is that patients are at a certain risk of suffering CAD, which correspond to different substrates. Focusing on nonCAD group, $\bar{\tau}_{e}=20.96 \pm 22.86 s$ and $\bar{\tau}_{r}=49.05 \pm 23.64 s$, where $\bar{\tau}$ denotes the mean values. This is in agreement with [12], where the mean time delays of QT interval adaptation for HR step-like accelerations and deceleration reached values of $\bar{\tau}_{e}=34.79 \pm 13.63 \mathrm{~s}$ and $\bar{\tau}_{r}=48.40 \pm 25.70 \mathrm{~s}$, respectively, in control subjects performing postural changes.

\section{Conclusion}

QT hysteresis can be modeled as the time constant of the response of a HR step-like input to a first order system, and this study shows that it is possible to estimate this delay from a ramp-like HR manoeuver as stress test too, where QT responds with a delayed ramp-like to gradual HR changes. The time delay has been studied in exercise and recovery ramps independently, using a hyperbolic regression model to fit stationary $R R(n)$ to $Q T(n)$ dependency and obtaining a memoryless expected HRdependent QT series to be compared to the actual $Q T(n)$ series. The delay at the exercise ramp shows potential as marker for CAD risk, given its increase for higher risk patients. Interestingly, in a reverted behaviour, significant decreased delay $\tau_{r}$ is observed at the recovery phase. Fi- 
Table 1. Mean \pm standard error for HR (bpm) and QT(ms) of each window, and $\alpha, \beta$ and $\varepsilon_{\mathrm{rms}}$ (ms) for hyperbolic model.

\begin{tabular}{|c|c|c|c|c|c|c|c|c|c|}
\hline & $\mathrm{HR}_{W_{1}}$ & $\mathrm{QT}_{W_{1}}$ & $\mathrm{HR}_{W_{2}}$ & $\mathrm{QT}_{W_{2}}$ & $\mathrm{HR}_{W_{3}}$ & $\mathrm{QT}_{W_{3}}$ & $\alpha$ & $\beta$ & $\varepsilon_{\mathrm{rms}}$ \\
\hline nonCAD & $77.5 \pm 13.3$ & $0.36 \pm 0.03$ & $159.9 \pm 14.5$ & $0.25 \pm 0.02$ & $94.0 \pm 13.6$ & $0.34 \pm 0.03$ & $-0.08 \pm 0.01$ & $0.47 \pm 0.03$ & $4.6 \pm 3.1$ \\
\hline Low-risk & $71.2 \pm 14.9$ & $0.38 \pm 0.03$ & $142.6 \pm 23.2$ & $0.27 \pm 0.03$ & $83.9 \pm 15.8$ & $0.36 \pm 0.03$ & $-0.09 \pm 0.02$ & $0.48 \pm 0.04$ & $5.8 \pm 4.4$ \\
\hline Mild-risk & $74.1 \pm 11.4$ & $0.37 \pm 0.03$ & $128.1 \pm 19.7$ & $0.29 \pm 0.03$ & $81.7 \pm 14.7$ & $0.37 \pm 0.03$ & $-0.10 \pm 0.02$ & $0.50 \pm 0.04$ & $5.6 \pm 5.2$ \\
\hline
\end{tabular}
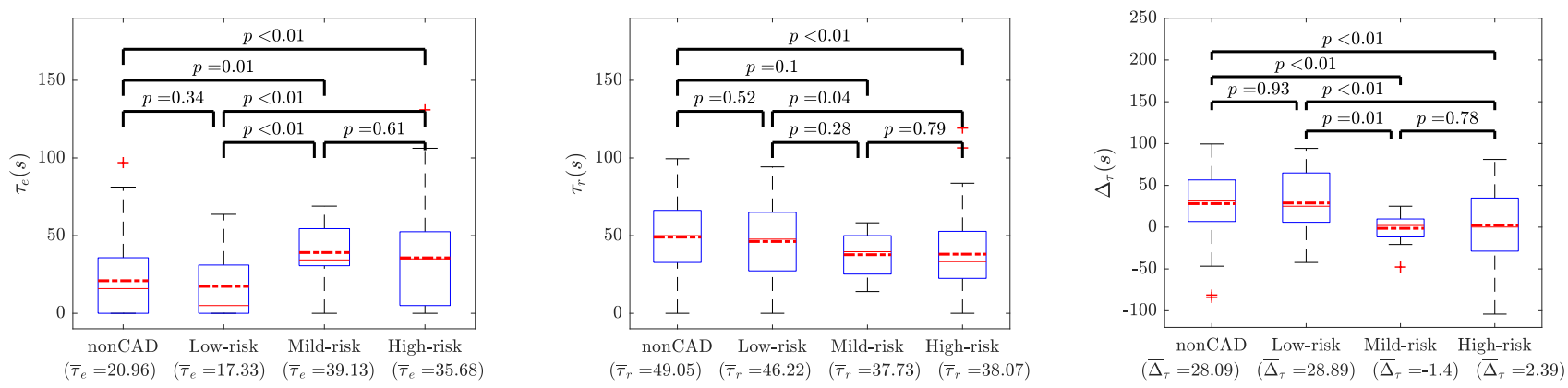

Figure 2. Box plots of the delay between estimated $\widehat{Q T}(n)$ and real $Q T(n)$ time series in exercise, $\tau_{e}$, and in recovery, $\tau_{r}$. $\Delta_{\tau}$ is the difference between recovery and exercise delays. The broken lines correspond to the mean values.

nally, the difference between delays at exercise and recovery shows well marked differences with CAD risk, being remarkably larger for nonCAD and low-risk patients and being much reduced when CAD risk increases.

\section{Acknowledgments}

This work was supported by projects ERC-StG 638284 (European Research Council), PID2019-105674RB-I00 and PID2019-104881RB-I00 (Ministerio de Ciencia e Innovación) and project LMP124-18 and reference group T39-20R (Aragón Government cofunded by FEDER 20142020 "Building Europe from Aragon"). Computations were performed using ICTS NANBIOSIS (HPC Unit at University of Zaragoza).

\section{References}

[1] Laguna P, Martínez JP, Pueyo E. Techniques for ventricular repolarization instability assessment from the ECG. Proceedings of the IEEE 2016;104(2).

[2] Pueyo E, Smetana P, Caminal P, De Luna AB, Malik M, Laguna P. Characterization of QT interval adaptation to RR interval changes and its use as a risk-stratifier of arrhythmic mortality in amiodarone-treated survivors of acute myocardial infarction. IEEE Transactions on Biomedical Engineering 2004;51(9).

[3] Pueyo E, Husti Z, Hornyik T, Baczkó I, Laguna P, Varró A, Rodríguez B. Mechanisms of ventricular rate adaptation as a predictor of arrhythmic risk. American Journal of Physiology Heart and Circulatory Physiology 2010;298(5).

[4] Porta A, Tobaldini E, Montano N. RT variability unrelated to heart period and respiration progressively increases dur- ing graded head-up tilt. American Journal of Physiology Heart and Circulatory Physiology 2010;298(5).

[5] Kligfield P, Lax KG, Okin PM. QT interval-heart rate relation during exercise in normal men and women: definition by linear regression analysis. Journal of the American College of Cardiology 1996;28(6).

[6] Ogata K. Modern Control Engineering. Instrumentation and controls series. Prentice Hall, 2010.

[7] Nieminen T, Lehtinen R, Viik J, Lehtimäki, et al. The Finnish Cardiovascular Study (FINCAVAS): characterising patients with high risk of cardiovascular morbidity and mortality. BMC cardiovascular disorders 2006;6(1).

[8] Castells F, Laguna P, Sörnmo L, Bollmann A, Roig JM. Principal component analysis in ECG signal processing. EURASIP Journal on Advances in Signal Processing ;2007.

[9] Martínez JP, Almeida R, Olmos S, Laguna P. A waveletbased ECG delineator: evaluation on standard databases. IEEE Transactions on biomedical engineering 2004;51(4).

[10] Killick R, Fearnhead P, Eckley I. Optimal detection of changepoints with a linear computational cost. Journal American Statistical Association 2012;107.

[11] Sampedro-Puente DA, Fernandez-Bes J, Szentandrássy N, Nánasi PP, Pueyo E. Time course of low-frequency oscillatory behavior in human ventricular repolarization following enhanced sympathetic activity and relation to arrhythmogenesis. Frontiers in Physiology 2019;10.

[12] Pueyo E, Malik M, Laguna P. A dynamic model to characterize beat-to-beat adaptation of repolarization to heart rate changes. Biomedical Signal Processing and Control 2008;

Address for correspondence:

Cristina Pérez (cperez@unizar.es)

Ed. Ada Byron, C/María de Luna 1, 50018 Zaragoza, Spain 